Discussion and conclusion There was not enough evidence from this work to suggest a switch, with the associated resource costs, to deliver the induction programme from faceto-face to VR or other pre-recorded media. However, given the positive response from students who had previously experienced VR, we have created a 360 degree tour of the hospice and induction programme to pilot using equipment on loan and evaluate its acceptability and effectiveness as a delivery method of induction at the hospice.

\section{THE PALLIATIVE A-E: AN ABCDE APPROACH TO ASSESSING AND MANAGING DYING PATIENTS USING SIMULATION TEACHING}

Michael Casey, Emma Husbands. Gloucestershire Hospitals Foundation Trust

\subsection{6/bmjspcare-2019-ASP.39}

Background $\mathrm{ABCDE}$ approaches are a proven and effective method of assessment in emergencies but could they be used in end of life care? This project developed a simulation teaching station for Foundation Programme Doctors to learn and practice an ABCDE style examination in the unconscious dying patient who cannot communicate symptoms verbally. The aim was to improve the confidence in this junior doctor cohort around assessing and managing dying patients.

Methods During trust teaching, a cohort of Foundation Doctors from Gloucestershire Hospitals NHS Trust were presented with a simulation case assessing an unconscious dying patient. One member from each group assessed the patient with other members contributing ideas. Ultimately the group was taught an ABCDE approach (a structured examination assessing Airway, Breathing, Circulatory, Disability and Exposure aspects, tailored to dying patients) and questioned on management options, with feedback throughout. A survey conducted before and after the teaching aimed to assess the cohort's confidence in assessing these patients.

Results The pre-intervention surveys demonstrated a lack of confidence in Junior Doctors in assessing dying patients both before and after graduation with 30 out of 30 and 28 out of 30 candidates feeling somewhat confident or less, respectively, partly attributed to a lack of teaching. Following the session, confidence in assessing these patients improved with 15 out of 20 feeling very or extremely confident and $100 \%$ of candidates finding the session and ABCDE technique useful.

Conclusions The use of an ABCDE approach to assessing the dying patient appears to improve the confidence Junior Doctors have with this skill. A simulation teaching session allowed for effective demonstration and practice of this tool. This method may lead to an improvement in symptom control in our dying hospital patients however, further research is needed.

\section{A QUALITATIVE STUDY EXPLORING HOW FOUNDATION DOCTORS FEEL THEIR UK UNDERGRADUATE MEDICAL EDUCATION HAS PREPARED THEM FOR CARING FOR THE DYING}

Rachael McDonald, Karen Groves, Julia Downing. Cardiff University, Queenscourt Hospice

10.1136/bmjspcare-2019-ASP.40
Background Newly qualified doctors working within the Foundation Programme are expected to care for dying patients, however many feel inadequately prepared for this. Given recent changes to the outcomes expected of medical graduates and national guidance surrounding the care of dying patients, there is a need to evaluate how UK graduates feel their training has prepared them to care for these patients.

Research question What are Foundation doctors' perspectives on how their UK undergraduate medical education has prepared them for caring for the dying?

Objectives

1. To investigate how Foundation doctors feel their UK undergraduate medical education has prepared them for caring for the dying.

2. To consider how undergraduate medical training in caring for the dying may be improved, from the perspective of Foundation doctors.

Methodology Qualitative data was collected by means of semistructured interviews with eight Foundation doctors who had experienced caring for dying patients since graduation. Thematic analysis was used to analyse the data.

Results Six themes were identified: 'The undergraduate course: what medical students are taught and what influences how they learn'; 'the role of the clinical team'; 'changing roles and practice'; 'preparedness to care for dying patients'; 'the culture that patients 'get better' and the realisation that they die'; 'recommendations for undergraduate training'.

Discussion All participants identified areas relating to the care of dying patients that they did not feel well prepared for. This suggests that current undergraduate training is not preparing graduating doctors adequately for this role. The theme of preparedness to care for dying patients can be subdivided into knowledge-based preparedness and emotional preparedness. Meaningful clinical contact appears to be integral to increasing knowledge-based preparedness. It should be recognised that caring for dying patients is often emotive; training surrounding dealing with this is required in order to avoid emotional distress and burnout.

\section{A UK PALLIATIVE TRAINEE RESEARCH COLLABORATIVE: NEW KNOWLEDGE THROUGH NETWORKING}

Charlotte Chamberlain, Guy Schofield, Sophie Hancock, Simon Etkind, Sara Robbins, Felicity Werrett, Hazel Coop, Rebecca Watson, Jonathan Koffman, Simon Noble. on behalf of the UK Palliative trainee Research Collaborative

\subsection{6/bmispcare-2019-ASP.41}

Background The first UK trainee research collaborative was established in 2006 and the majority (39/45) are surgical or anaesthetic based. Trainee collaboratives harness the resource of doctors in training for multicentre audit and research. Collaboratives have spearheaded quality improvement projects, audit, systematic reviews and recruitment for national and international studies. Collaboratives have also demonstrated increased trainee research exposure over time, with trainee involvement in design, ethics, data analysis, and manuscript preparation.

Palliative medicine research is relatively underfunded, and trainee research exposure varies significantly across rotations. Our small specialty has much to gain from the 